\title{
DETERMINATION OF ALL CLASSES OF POSITIVE QUATERNARY QUADRATIC FORMS WHICH REPRESENT ALL (POSITIVE) INTEGERS ${ }^{1}$
}

\author{
MARGARET F. WILLERDING
}

1. Introduction. It was apparently known to Diophantus and first proved by Lagrange $[8]^{2}$ that the form $x^{2}+y^{2}+z^{2}+u^{2}$ represents all positive integers. Examples of other integral forms

$$
\Phi=a x^{2}+b y^{2}+c z^{2}+d u^{2}
$$

which represent all positive integers were first obtained by Jacobi [4], Liouville [9], and Pepin [13]. Ramanujan [14] proved that there are only 54 sets of positive integers $a, b, c, d$, such that (1.1) represents all positive integers. Dickson called all such forms universal [3].

Among the 54 universal forms of type (1.1) the form $x^{2}+2 y^{2}+4 z^{2}$ $+14 u^{2}$ has the largest determinant, 112 [1].

It is of interest to ask what would be the largest determinant of the universal form if we should allow cross product terms. If we should consider forms whose cross product terms may have odd coefficients then the value of the determinant may not exceed 861 [17], and is of that order of magnitude. If, however, we should consider only the so-called "classic" forms, that is, forms whose cross product terms have necessarily even integral coefficients, then we find the value of the largest determinant of such universal forms is, remarkably enough, again 112 [15].

The number of universal forms without cross products, whose coefficients are arranged in the order of increasing magnitude, is equal to 54 [2]. Since such forms are reduced forms we may rephrase this result to say that the number of classes of forms which are equivalent to a form without cross products is equal to 54 .

Pursuing this idea further we may ask what is the number of classes of classic universal forms, or extending still farther the set of admissible forms, we may attempt to determine the number of universal classes of non-classic integral forms. In the last case this number is very large as one can see from the work of Morrow [10]. However since in the case of classic forms the upper bound for the determinant is small (112), one should expect that the number of uni-

Presented to the Society, September 3, 1947; received by the editors June 27, 1947.

1 This paper is a portion of a dissertation submitted in partial fulfillment of the requirements for the degree of Doctor of Philosophy at St. Louis University.

2 Numbers in brackets refer to the bibliography at the end of the paper. 
versal classes of classic forms would not be large. It is of interest, therefore, to determine this number exactly.

Employing an extension of the method of Ramanujan we find:

ThEOREM 1. There are exactly 178 classes of universal classic positive quaternary quadratic forms $[18] .^{3}$

2. General theory. It was shown in [15] that every classic universal quaternary quadratic form is equivalent to a quadratic form

$$
f=x^{\prime} A x=x_{1}^{2}+\sum_{i, j=2}^{4} a_{i j} x_{i} x_{j}=x_{1}^{2}+\psi\left(x_{2}, x_{3}, x_{4}\right)
$$

where the ternary form $\psi\left(x_{2}, x_{3}, x_{4}\right)$ is a reduced positive ternary form of determinant less than or equal to 112 .

Jones' table [6] of these forms shows that there are 1046 such forms and gives the first rough upper bound for the number of classes of classic universal quaternary quadratic forms.

To obtain the exact count of the number of universal classes one must examine each one of these 1046 forms. Many of these forms are eliminated immediately by showing that they do not represent some small integer. Forms which pass these tests are scrutinized more carefully and submitted to more powerful number theoretic tests for proving universality. To prove the remaining forms universal it was necessary to use three somewhat different methods.

3. Tests for universality: Method 1. The first method proceeds essentially as follows. We complete the squares in the form (2.1) by multiplying $f$ by a suitable integer $n$ in such a way that if $F=n f$ represents all positive integral multiples of $n$, then $f$ represents all positive integers.

Let $S$ be the regular or almost regular ternary section of $F$ which represents all positive multiples of $n$ not of a certain form $Q, Q$ being the arithmetic progression associated [2, 6] with $S$. We write $F=S+c x_{k}^{2}$. Now if it can be shown that for every integer of the form $Q, S$ represents $Q-c x_{k}^{2}$ for a suitable value of $x_{k}$ and the given value of $c$, then $F$ represents $Q$ and therefore every positive integral multiple of $n$. Hence $f$ is universal.

4. Tests for universality: Method 2. In case the form $\psi$ in (2.1) is a regular ternary form [16] one may proceed very much as in the first method. Since $\psi$ is regular it represents all positive integers not

\footnotetext{
${ }^{3} \mathrm{~A}$ table of these forms may be found in a more complete version of this dissertation deposited in the American Mathematical Society Library.
} 
of a certain form $Q, Q$ being the arithmetic progression associated $[2,6]$ with $\psi$. Now if it can be shown that for every integer of the form $Q, \psi$ represents $Q-x_{k}^{2}$, for a suitable value of $x_{k}$, then $f$ represents $Q$ and all positive integers.

5. Tests for universality: Method 3. In some forms due to the irregularity of $\psi$ or the ternary sections of the corresponding $F$ form, or because the representation of positive integral multiples $a \cdot n$ of $n$ by $F=n f$ does not insure an integral representation of $a$ by $f$, universality is tested by a different method.

In this method two ternary forms $S_{1}$ and $S_{2}$ are obtained by the substitution $x=H y$ where $H=\left[h_{i j}\right], i=1,2,3,4 ; j=1,2,3$.

By referring to a table giving the generic characters of such ternary forms [11] or by computing the generic characters in the cases of the improperly primitive forms it is possible to determine whether or not the ternary sections $S_{1}$ and $S_{2}$ are in a genus of one class. Since such forms are regular [7], it may be concluded that $S_{1}$ and $S_{2}$ are regular.

The progressions associated with $S_{1}$ and $S_{2}$ are compared [16]. If the integers excluded by the progressions for $S_{1}$ are represented by $S_{2}$ and those excluded by the progressions associated with $S_{2}$ are represented by $S_{1}$ it follows that $f$ represents every positive integer.

\section{BiBLIOGRAPHY}

1. L. E. Dickson, History of the theory of numbers, vol. 3, Quadratic and higher forms, New York, Stechert, 1934. 1939.

2. - Modern elementary theory of numbers, University of Chicago Press,

3. - Quaternary quadratic forms representing all integers, Amer. J. Math. vol. 49 (1927) pp. 39-56.

4. C. G. J. Jacobi, Werke, vol. 6, pp. 281-302.

5. B. W. Jones, $A$ table of Eisenstein-reduced positive ternary quadratic forms of determinant $\leqq 200$, Bulletin No. 97 of the National Research Council, 1935.

6. B. W. Jones and G. Pall, Regular and semi-regular positive ternary quadratic forms, Acta Math. vol. 70 (1939) pp. 165-195.

7. B. W. Jones. The regularity of a genus of positive ternary quadratic forms, Trans. Amer. Math. Soc. vol. 33 (1931) pp. 111-124.

8. J. L. Lagrange, Oeuvres, vol. 3, 1869, pp. 189-201.

9. J. Liouville, Journal de Mathématiques (2) vol. 1 (1856) p. 230.

10. D. C. Morrow, Determination of all quaternary quadratic forms which represent every positive integer, Unpublished Thesis, University of Chicago, 1928.

11. C. Neville, On genera of positive ternary quadratic forms of determinant $\leqq 200$ containing a single class, Unpublished Thesis, St. Louis University, 1940.

12. G. Pall, Note on almost-universal forms, Bull. Amer. Math. Soc. vol. 46 (1940) p. 291.

13. T. Pepin, Atti Accademia Pontificia dei Nuovi Lincei vol. 38 (1884-1885) pp. 171-196. 
14. S. Ramanujan, On the expression of a number in the form $a x^{2}+b y^{2}+c z^{2}+d v^{2}$, Proc. Cambridge Philos. Soc. vol. 19 (1916) pp. 11-12.

15. A. E. Ross, On a problem of Ramanujan, Amer. J. Math. vol. 68 (1946) pp. $29-46$.

16. - On representation of integers by indefinite ternary quadratic forms of quadratfrei determinant, Amer. J. Math. vol. 55 (1933).

17. —, Manuscript.

18. M. F. Willerding, Determination of all classes of positive quaternary quadratic forms which represent all (positive) integers, Dissertation, St. Louis University, 1947.

WASHINGTON UNIVERSITY 